Cahiers de philosophie de l'université de

Levinas : au-delà du visible

\title{
La phénoménologie et son double. Le son parle, la parole sonne
}

Danielle Cohen-Levinas

\section{(2) OpenEdition}

1 Journals

Édition électronique

URL : https://journals.openedition.org/cpuc/803

DOI : $10.4000 /$ cpuc. 803

ISSN : 2677-6529

Éditeur

Presses universitaires de Caen

Édition imprimée

Date de publication : 31 juillet 2012

Pagination : 85-100

ISBN : 978-2-84133-410-0

ISSN : 1282-6545

\section{Référence électronique}

Danielle Cohen-Levinas, « La phénoménologie et son double. Le son parle, la parole sonne », Cahiers de philosophie de l'université de Caen [En ligne], 49 | 2012, mis en ligne le 07 juin 2018, consulté le 31

janvier 2023. URL : http://journals.openedition.org/cpuc/803 ; DOI : https://doi.org/10.4000/cpuc.803

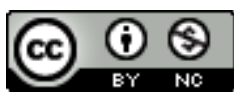

Creative Commons - Attribution - Pas d'Utilisation Commerciale 4.0 International - CC BY-NC 4.0 https://creativecommons.org/licenses/by-nc/4.0/ 


\section{La phénoménologie et son double. Le son parle, la parole sonne}

Le silence n'est pas réalité - mais intervalle.

Levinas $^{1}$

À peine l'oreille en a-t-elle été frappée [le son], qu'il rentre dans le silence.

Hegel $^{2}$

C

OMMENÇONS PAR UN ÉLÉMENT qui dans sa manifestation signifie l'expérience d'une subjectivation hors de soi, se situant au-delà du langage de l'Être, en dehors de l'expérience de la responsabilité pour l'Autre. En 1944, Levinas écrit dans les Carnets de captivité: «La sonorité du son: trois caractéristiques: 1) il se penche sur l'avenir ; 2) il s'approfondit en luimême; 3) il est un éclat ${ }^{3}$. Quelques années plus tard, en 1948, lors d'une conférence intitulée "Parole et Silence» ${ }^{4}$, Levinas revenait sur la question de la sonorité et l'éclat du son par opposition au monde de la lumière:

1. E. Levinas, Carnets de captivité, in Carnets de captivité, suivi de Écrits sur la captivité et Notes philosophiques diverses (= Euvres 1), R. Calin et C. Chalier (éd.), Paris, Grasset - IMEC, 2009, p. 133.

2. G.W.F. Hegel, Esthétique, traduit de l'allemand par C. Bénard sur la première édition des Cours d'esthétique (G.W.F. Hegel's Vorlesungen über die Aesthetik), traduction revue par B. Timmermans et P. Zaccaria, vol. II, Paris, Librairie générale française (Le Livre de poche), 1997, p. 322.

3. Carnets de captivité, p. 145.

4. Cette conférence est datée des 4 et 5 février 1948 et a été prononcée en deux séances à l'invitation de Jean Wahl au Collège philosophique. Voir E. Levinas, Parole et Silence et autres conférences inédites au Collège philosophique (= Euvres 2), R. Calin et C. Chalier (éd.), Paris, Grasset - IMEC, 2011, p. 69-104. 
Dans son retentissement. Dans son être même, le son est éclat. Ou pour le dire, d'une façon qui fait davantage ressortir son caractère social - le son est scandale 5 .

Si loin que l'on avance dans la lecture des textes d'Emmanuel Levinas, y compris des textes les plus tardifs, nous ne trouvons trace d'une philosophie du son aussi éloquente d'un rapport à l'intentionnalité qui ne se limite pas à la structure noético-noématique. Le son appartiendrait à une catégorie de la donation du sens (Sinngebung), comme une singularité originaire désignant les caractéristiques de l'existence sensible. Toutes les données du son sont des formes de don que Levinas relie dans les Carnets de captivité au thème de l'approche, qu'il essaie de thématiser dans les esquisses de son roman $^{6}:$ "Les êtres vous approchent d'abord avec leur nuage d'atmosphère, comme une musique. Comme un leitmotiv wagnérien».

La musique dont parle Emmanuel Levinas ici, dont nous savons qu'elle n'occupe pas dans son œuvre une place centrale, en dehors de quelques évocations et références restreintes, est abordée comme une disposition de l'être à s'accorder avec un autre être. Le mot "atmosphère» n'est pas fortuit, pas plus que la référence wagnérienne, d'aucuns penseront qu'elle est inattendue, alors même qu'elle nous semble renvoyer indirectement à Nietzsche. Il stipule la dimension de Stimmung ${ }^{7}$, que l'on traduit souvent par tonalité ou atmosphère, et dont l'ancrage dans le vocabulaire musical se fait depuis le mot Stimmen, qui contient une dimension d'appel, laquelle requiert une dimension temporelle qui est le propre du son. En termes phénoménologiques, nous pourrions dire que Levinas fait du corps sonore une chair consonante avec le sensible, instituant entre le monde et le moi un passage, qui précéderait le discours ou qui viendrait surseoir à sa défaillance. Il ouvrirait et creuserait les écarts de la parole plutôt qu'il ne leur substituerait une plénitude et une harmonie mondaines. Or le figural, au sens précisément du leitmotiv wagnérien, libère une dimension sensible qui est encore captive de la forme linguistique. Le leitmotiv wagnérien n'est pas une simple métaphore esthétique. Il structure la métaphore de l'existence telle que Levinas souhaiterait la raconter dans son roman:

Une existence où il n'y a pas d'enracinement dans la terre mais où la vie se passe au milieu de courants chauds ou froids, d'émotions. Telle figure

5. «Parole et Silence», p. 9o.

6. Il s'agit du roman que Levinas a commencé en captivité, intitulé Eros ou plus vraisemblablement Triste opulence.

7. Il existe une œuvre de Karlheinz Stockhausen intitulée Stimmung (1968) pour six chanteurs et six microphones. 
vous ramène à telle région, vous plonge dans tel courant. L'existence comme installée dans une région où passent ces effluves ${ }^{8}$.

Cette conception du son comme figure musicale n'est pas distincte du mot et de l'énoncé aristotélicien comme ce qui serait une voix composée et signifiante. D’ailleurs le signe délié du sens correspond exactement à ce qu'Aristote nomme voix (phône) et que les Allemands nommeront Stimmung. L'existence comme motif wagnérien est à entendre comme une pure composition ou émergence d'une voix qui tantôt est recouverte par la dimension du sens, tantôt l'interrompt. Levinas trahit encore dans ses Carnets de captivité sa proximité avec La philosophie de l'esprit (1805), où Hegel fait entrer la voix de l'art dans la présence à soi de l'esprit. Levinas ne dit rien d'autre que ce qui se disait à l'époque d'Iéna. C'est à l'art musical que revenait d'intérioriser l'art en un pur entendre de l'esprit. Le paradoxe wagnérien est précisément que trop de figures tuent la figure. En faisant du leitmotiv wagnérien une métaphore de désenracinement, Levinas situe le son comme étant au commencement de l'intériorisation de l'esprit qui cherche à s'entendre lui-même. À y regarder de plus près, on constate que Levinas, dès les Carnets de captivité, tente de penser l'expérience du son comme force de relance, comme le moment névralgique où ce qui s'éprouve avec l'événement du son renvoie à la traduction d'un «dedans» "en dehors", de part et d'autre d'un retentissement logé dans la vibration même du son dont la vocation est de se temporaliser dans la communication avec autrui. Dans ce cas précis, le son se tient au seuil du dedans et du dehors, ni complètement attaché ni complètement détaché de l'esprit. Il est, écrit Levinas,

[...] essentiellement ce qui remplit le temps. Il est symbole en tant qu'éclat. Ce n'est pas pour se communiquer qu'on parle mais c'est parce qu'on se sert du son qu'on se communique. Je veux dire: si le son sert à la communication ce n'est pas en tant qu'il est objet extérieur qu'on remarque et à partir duquel on recrée en soi la pensée dont il procède. Le son c'est l'accomplissement de la communication. C'est la communication qui fait pousser le cri - qui nous introduit dans autrui - ce n'est pas grâce au cri que la communication s'établit seulement. Cette place du cri par rapport à la communication, c'est précisément la voix 9 .

Qu'en est-il de cette "communication" dont Levinas ambitionne dès les Carnets de captivité, puis dans la conférence "Parole et Silence», de déterminer une signification qui ne se limite pas à une problématique ontologique,

8. Carnets de captivité, p. 102.

9. Ibid., p. 145-146. 
mais qui annonce, à l'instar de l'œuvre d'art se situant pour Levinas en 1948 «en dehors de l'être au monde», ne se donnant pas "pour un commencement de dialogue $»^{10}$, un rythme propre, une dimension sensible non résiduelle, une intentionnalité dont l'appréhension ne neutralise plus la position d'objet opérée par la perception. C'est contre la limitation husserlienne d'un certain registre d'intentionnalité que Levinas nous semble postuler dans «Parole et Silence» pour une doublure qui n'est plus la conséquence d'une essence révélée dans la lumière, mais qui provient d'un dérangement n'ayant plus rien à voir avec la qualité de la chose, ni avec la relégation du sensible dans un arrière-fond de la perception. La phénoménologie du son telle que la conçoit Levinas répond à une logique tautologique qui est une mise en abyme de l'intentionnalité comme mise en lumière adéquate de l'objet idéal qui en serait le corrélat. C'est pourquoi Levinas peut dire: «Le son double toutes les manifestations de la chose $»^{11}$. Il nous parait essentiel de rappeler que «La réalité et son ombre» fut publiée juste quelques mois après que Levinas ait prononcé la conférence «Parole et Silence» ${ }^{12}$. Il est donc important d'insister sur un point: la réflexion sur la phénoménologie du son se démarque aussi de la réception de la phénoménologie par la pensée française de l'époque, en particulier de la Phénoménologie de la perception ${ }^{13}$ de Merleau-Ponty. À ce titre, Levinas cherche à redonner à la question de la sensation et du sensible une assise phénoménale synonyme d'un côté de «dégagement» pour l'œuvre d'art, d'instant immobilisé qui n'a prise ni sur un passé ni sur un avenir, et de l'autre, pour le son en particulier, de «dérangement» et de déversement dans le temps. Si le son peut doubler toutes les manifestations de la chose, c'est précisément qu'il n'est pas un arrêt du temps ou, comme dans toute œuvre d'art, "son retard sur luimême ${ }^{14}$. L'instant du son, plus exactement son instantanéité, n'est pas rebelle à l'avenir. Le son ne raconte pas la plasticité d'une histoire, comme le ferait l'œuvre d'art ou le mythe. Levinas a cette formule extraordinaire pour distinguer une certaine fixité de l'image, de la couleur et de la lumière et l'ébranlement par le son. L'une répond à un processus de «dégradation

10. "La réalité et son ombre», in Les imprévus de l'histoire, Montpellier, Fata Morgana, 1994, p. 123-148.

11. "Parole et Silence», p. 91.

12. Le texte «La réalité et son ombre» a été publié dans Les Temps Modernes en novembre 1948 (numéro 38). La réflexion sur l'art qu'il déploie dans cet article est précédée d'un chapeau préliminaire rédigé sans doute par Merleau-Ponty (p. 769-770), dans lequel ce dernier prend clairement ses distances avec la réflexion de Levinas en lui reprochant de n'avoir examiné qu'à moitié l'engagement de Sartre avec la littérature. "Parole et Silence» a été prononcé en deux fois, les 4 et 5 février 1948.

13. Paris, Gallimard, 1945.

14. «La réalité et son ombre», p. 138. 
ou d'une érosion de l'absolu» ${ }^{15}$; par l'autre $«[\ldots]$ l'absolu s'impose $»^{16}$. Dans les deux cas, œuvre d'art et phénoménologie du son, nous sommes tout prêts du livre publié en 1947, De l'existence à l'existant, dont une large part fut rédigée pendant la captivité. L'œuvre d'art, placée au registre de l'«exotisme», se trouve dans le voisinage d'une "existence sans monde» que Levinas désigne par il y a. Venant du dehors, le son a pour vocation d'échapper à la description heideggérienne de l'être-au-monde sans pour autant renoncer à l'idée qu'il est «l'analogon de l'être ${ }^{17}$.

On ne peut aborder la place et la fonction qu'occupe la phénoménologie du son dans les textes posthumes d'Emmanuel Levinas, qu'il soit question des Carnets de captivité ou de la conférence "Parole et Silence», sans interroger l'enseignement phénoménologique de Heidegger centré sur l'être-au-monde. Si l'analytique heideggérienne souligne que le Dasein est à la fois dans la vérité et dans la non-vérité, écartelé entre la polarité du propre et de l'impropre, Levinas cherche à sortir de la cooriginarité de l'être et de la vérité. Ce que Levinas appelle «la sonorité du son $»^{18}$, dans son plein retentissement, dans son plein éclat, dans "son être même» ${ }^{19}$, ne saurait enfermer l'être dans le solipsisme ou ne saurait être d'une problématique ontologique qui ne peut se déborder elle-même.

On notera donc que la tâche que s'assigne Levinas est d'abord d'inspiration heideggérienne. Dans les Carnets de captivité, il écrit:

L'Être n'est pas seulement un verbe - c'est le Verbe. Distinction entre verbe et action, verbe et mouvement, verbe et devenir. Tout cela est l'analogon de l'être. Devenir le plus rapproché - mais habituellement conçu en vue du terme. - Ce qui n'est pas analogon - mais être même - c'est le fait qu'il est verbe - Le verbe c'est le son - Le son en tant que retentissement - vibration - ce qu'il y a d'action dans la vibration - se maintenir - une certaine amplitude D'où différence entre voir - comprendre et entendre $-{ }^{20}$.

En d'autres termes, le Verbe sonne. Levinas n'utile pas le mot «langage», ou «parole», sur le mode die Sprache spricht comme le fait Heidegger dès les années cinquante, ce qui reviendrait à dire, der Klang klingelt. La tournure tautologique est bien là et avec elle une certaine qualité apophantique. Le son en tant que retentissement libère une vibration qui est Verbe, comme s'il était chargé d'une dimension herméneutique. Nous comprenons à travers

15. Ibid., p. 136.

16. «Parole et Silence», p. 91.

17. Carnets de captivité, p. 166. Analogon est souligné par Levinas.

18. "Parole et Silence», p. 90.

19. Ibid.

20. Carnets de captivité, p. 166. 
le retentissement du son ce qui relève déjà du Verbe, se donnant dans une forme indéterminée, anté-prédicative. Mais le son peut-il être pensé à partir de lui-même, dans l'identité à soi, se prenant pour son propre référent, pleinement tautologique? En termes purement acoustiques et dans la mesure où le son est un phénomène ondulatoire, il renvoie nécessairement à luimême, tout en se propageant dans l'espace de manière périodique, à savoir sous une forme qui se répète à l'identique dans le temps, ou aléatoire, ce qui signifie que le son n'a pas de caractéristiques périodiques. De sorte que tous les sons peuvent s'appréhender soit dans l'espace temporel, soit dans l'espace fréquentiel. Autrement dit, soit notre sensation auditive perçoit le son comme un signal statique, une vibration stable dans le temps qui demeure enfermée dans sa propre enveloppe «comme un son qui ne serait audible que dans son propre écho ${ }^{21}$, soit elle le perçoit comme un signal évolutif qui se temporalise et dont le spectre ${ }^{22}$ est «l'accomplissement de la communication ${ }^{23}$. Dans le premier cas, le son, comme un corps ou hypostase, se pose, vibre et retombe sur soi. L'objet son, une fois émis, est comme ramené en arrière dans un mouvement instantané où l'arsis et la thesis sont constitutives d'un unique mouvement instantané qui ne doit rien au temps. Dans le deuxième cas, le son ne se contente pas de sonner, il endure une durée qui l'arrache à une extinction qui ferait du surplace en lui substituant ce que l'on appelle une vibration continue, tendue vers l'avenir. Nul besoin d'insister sur la résonance philosophique de ce bref descriptif. On entrevoit ici ce que Levinas tente d'esquisser : le passage d'une problématique ontologique de la phénoménologie à une problématique éthique de la phénoménologie; le passage d'une «lumière qui revient " ${ }^{24}$ à un son "qui se refait» ${ }^{25}$. La signification du son viendrait interrompre sa matérialité, comme une tentative de passer outre l'effectivité événementielle inapte à engendrer un processus temporel. Il faut suivre le cheminement de la pensée de Levinas pour comprendre pourquoi dans «La réalité et son ombre» il insistera tant sur la musicalité de l'image et sur le rythme propre qu'elle requiert. Je ne pense pas qu'il faille en déduire une pensée plus ou moins explicite de la musique. Ce qui me paraît exemplaire et très novateur pour l'époque, c'est la manière dont Levinas défait la relation entre son et rythme, comme si en arrachant le rythme à son timbre, le son s'émancipait de l'anonymat. L'histoire de la musique occidentale s'est construite sur ce

21. Humanisme de l'autre homme, Montpellier, Fata Morgana, 1972, p. 16.

22. Le spectre d'un signal sonore est composé des différentes notes dites «sons purs» que contient un son. Ces sons purs sont appelés «partiels».

23. Carnets de captivité, p. 146.

24. Ibid., p. 167.

25. Ibid. 
rapport insécable du rythme et du son, de sorte que l'intention de l'oreille tendue vers la musique va directement au rythme tout en étant touchée par le son. Le rythme s'impose à celui qui l'écoute. Il s'impose par-delà l'écoute elle-même comme ce qui viendrait forcer notre participation:

Mais ils s'imposent à nous sans que nous les assumions. Ou plutôt, notre consentement à eux s'invertit en participation. Ils entrent en nous ou nous entrons en eux, peu importe. Le rythme représente la situation unique où on ne puisse parler de consentement, d'assomption, d'initiative, de liberté - parce que le sujet en est saisi et emporté ${ }^{26}$.

Non seulement le rythme est relié à la musique, mais il relie également la conscience de soi à l'intériorité subjective. Sur ce point précis, Levinas est en consonance totale avec ce que dit Hegel du rythme musical comme vecteur d'une signification qui serait en résonance avec l'organisation interne de la subjectivité. En définitive, ce n'est pas le son qui est le paramètre le plus expressif de la musique, mais le rythme, car ce dernier est centré sur le sujet lui-même, d'où le lien étroit que Hegel établit entre le temps et le moi:

[...] le moi n'est pas une continuité indéterminée, la durée sans fixité; il n'a d'identité véritable qu'autant qu'il rassemble les moments épars de son existence et fait un retour sur lui-même. Ce n'est qu'en se repliant sur soimême et en se prenant pour objet qu'il existe pour lui-même, et que, par ce rapport avec lui-même, il devient sentiment de soi, conscience de soi $^{27}$.

C'est donc sur le sol de ce qu'il cherche à dépasser que Levinas inscrit sa phénoménologie du son. Ce que la «sonorité du son» fait résonner, c'est précisément ce qui ne change pas, c'est-à-dire une ontologie doublée avec Hegel d'une dialectique. En affirmant, comme il le fait dans les Carnets de captivité, que «[...] si le son sert à la communication ce n'est pas tant qu'il est objet extérieur qu'on remarque et à partir duquel on recrée en soi la pensée dont il procède ${ }^{28}$, Levinas ne fait qu'entériner l'idée que le son / rythme identifie la conscience avec la subjectivité la plus intime: «[...] c'est quelque chose qui appartient au moi, qui est transporté de lui au temps pour sa propre satisfaction ${ }^{29}$. Du coup, la présence du son est amarrée au sujet transcendantal, faisant signe à l'idéalisme absolu. Mais pour Levinas, fin lecteur de Hegel, le son, comme tout ce qui est absolu, fait signe vers quelque chose qui se situe au-delà de lui. Si l'esthétique musicale de Hegel

26. "La réalité et son ombre», p. 128.

27. Hegel, Esthétique, p. 347.

28. Carnets de captivité, p. 145.

29. Hegel, Esthétique, p. 349. 
présuppose l'identité du sujet et de l'objet son comme manifestation de l'immédiateté première, la phénoménologie du son de Levinas s'éloigne sans y renoncer à cette absence de contradiction selon laquelle l'expression musicale engloberait la totalité de la conscience, ferait vibrer les cordes de l'âme et la mènerait au silence.

\section{«L'oreille qui se fatigue à scruter le silence ${ }^{30}$}

Il nous faut revenir au son comme «scandale " ${ }^{31}$, comme ce qui vient déchirer - «crever» dit Levinas dans les Carnets de captivité - le silence:

Le son - en tant qu'éclat et symbole. Il y a des instruments qui n'ont pour but que le son en tant que tel: le tambour (avec le qqch. d'angoissant qu'il amène) et surtout la cloche qui crève le silence et qui remplit l'espace de quelque chose qui vient de là-bas ${ }^{32}$.

Arrêtons-nous un instant sur le motif de la cloche et sur l'expression «[...] qui vient de là-bas». La cloche est l'instrument qui en captivité battait le rappel, le rassemblement des prisonniers. Elle ne signifiait pas un renversement de la position solipsiste du son à une position où ce dernier serait expulsé de son essence de son. Cependant l'attention portée au son de la cloche est remarquable en raison du «son pur» ${ }^{33}$ qui n'est ni métaphorique ni allégorique ni tautologique: «Il n’est pas musique, mais il n'est pas simple signe. Le son dans sa sonorité même» ${ }^{34}$.

Comment entendre ce son, «dans sa sonorité même»? Le son de la cloche a ceci de particulier qu'il contient une charge d'inharmonicité35 très élevée comparativement aux autres instruments. L'inharmonicité de la cloche est exemplaire car elle peut aller jusqu'à produire plusieurs hauteurs que l'oreille perçoit simultanément. En produisant plusieurs hauteurs, elle installe des battements propres à chacune d'entre elles, lesquelles entrent en déphasage avec le son fondamental et provoquent un écart temporel, irréductible entre «la sonorité même du son ${ }^{36}$ et «le son dans sa sonorité

30. Carnets de captivité, p. 196.

31. "Parole et Silence», p. 90.

32. Carnets de captivité, p. 152.

33. Ibid.

34. Ibid.

35. En acoustique musicale, le phénomène d'inharmonicité s'oppose à l'harmonicité. Cela signifie que les composantes d'un son musical ne sont pas liées à des fréquences comme multiples entiers du son fondamental, alors que l'harmonicité d'un son signifie que les harmoniques sont en fréquence des multiples entiers du son fondamental, exactement comme dans le modèle mathématique d'un phénomène périodique.

36. «Parole et Silence», p. 90. 
même ${ }^{37}$. Ce qui est «crevé» l'est en vertu des propriétés disjonctives des sons inharmoniques, tout l'inverse d'un son qui maintiendrait la transparence de par ses propriétés conjonctives, dont la synthèse additionnelle des fréquences ne fait que renforcer la plénitude du son fondamental. Aussi, avec les sons inharmoniques de la cloche, avons-nous déjà en soi un modèle de clignotement ostensible et audible du va-et-vient entre la subjectivité de l'en-soi qui demeure rivée à soi et une subjectivité altérée par le jeu des battements désynchronisés de leur socle. Levinas perçoit très bien la différence. Ce qui est atteint par le son de la cloche, ce n'est précisément pas l'unité de ce qui sonne et qui en sonnant fait retentir un contenu donné par une forme "qui lui prête un sens » ${ }^{38}$, c'est une unité (le son fondamental) fissurée en de multiples unités qui ne sont reliées entre elles par aucune synthèse d'entendement. Le paradoxe de la réflexion de Levinas sur le son est qu'elle ne vise en rien à établir la relation de l'objet à penser. $\mathrm{Au}$ fond, à partir de la réduction husserlienne au son pur, par analogie au moi pur, Levinas situe ses méditations aphoristiques sur le son non pas sous la forme d'une élaboration théorique mais plutôt comme Selbstbesinnung. Toutefois, au lieu d'opérer un retour sur soi de la pensée du son comme organon se rapportant à lui-même, celle-ci mettrait en défection la subjectivité comme réflexion de la réflexion, ou encore comme sonorité de la sonorité ne signifiant plus un retour à des modalités acoustiques régi par le son lui-même. Avec le son de la cloche, la sonorité du son s'échappe du noyau dur, de la totalisation synchronisante et synthétisante. Sans pour autant s'attarder sur le son de la cloche, Levinas revient une fois de plus dans «Parole et Silence» sur la distinction entre lumière et son:

[...] l'essence du son est une rupture. Non pas la rupture dans le monde de la lumière et du silence - où la rupture découvre une continuité plus profonde, où la déchirure est sous-tendue par la continuité et l'universalité de l'espace. Mais rupture pure qui ne conduit pas à quelque chose de lumineux mais qui fait sortir de la lumière. En tant que qualité sensible, en tant que phénomène, le son est lumière; mais c'est un point de lumière où le monde éclate, où il est débordé. Ce débordement de la qualité sensible par elle-même, son incapacité de tenir son contenu - c'est la sonorité même du son ${ }^{39}$.

Comment ne pas entendre dans ses propos les tâtonnements déjà consignés dans les Carnets de captivité de ce que Levinas appelait «Contribution à ma théorie de la lumière $4^{\circ}$ ? Dans ce texte, il explique que ce qui fait du

37. Carnets de captivité, p. 152.

38. "Parole et Silence», p. 90.

39. Ibid., p. 90.

40. Carnets de captivité, p. 147. 
monde un soi, ce n'est pas la subjectivité de la sensation, mais sa luminosité. C'est le privilège de la représentation qui pour lui est synonyme de maîtrise et de souveraineté, une subjectivation qui ne vient pas du dehors et dont Levinas, dans un article intitulé «La ruine de la représentation ${ }^{41}$, montrera qu'elle n'est plus tributaire de la conscience:

La transparence du monde éclairé - l'espace - ce n'est rien et c'est tout. Transparence - rien ne s'oppose - il n'y a rien et cependant c'est l'être. Contribution à ma théorie de la lumière - où c'est moi-soi et où le soi devient monde et n'est que soi cependant. Marquer l'opposition de cette théorie à la théorie du solipsisme tiré de la relativité des sensations - de la composition du monde à partir de sensations subjectives ${ }^{42}$.

Avec la question du son comme prototype de ce qui pour réapparaître doit être reproduit de manière toujours nouvelle, à l'instar de la lumière qui revient à l'identique et jamais autrement, Levinas ne se contente pas de critiquer l'idée que toute conscience est conscience de quelque chose et que tout objet appelle et suscite la conscience par laquelle son être apparaît à la lumière et resplendit, il retourne l'intentionnalité husserlienne contre ellemême en montrant comment elle instaure un décrochage entre moi-soi et l'autre, faisant en sorte que l'intentionnalité objectivante qui est le propre de la représentation soit précisément minée de l'intérieur. Lorsque Levinas dit que le son est lumière, «mais c'est un point de lumière où le monde éclate » 43 , il parle prioritairement me semble-t-il d'une intentionnalité signifiante d'un autre éclatement, celui de la conscience. Or c'est la phénoménologie husserlienne qui permit à Levinas ce geste de retournement, car en accordant à la sensation un statut inégalable, une place primordiale, Husserl ouvre la voie à la transcendance de l'intentionnalité comme présence au monde. Il y a là en effet une rupture avec la pensée de la représentation. Impossible de dire par exemple si le son de la cloche - son pur comme le souligne Levinas - est musique ou simple signe. Il est dans sa sonorité même, et à ce titre il devient une figure d'équivocité: un éclat et un symbole que Levinas ne tardera pas, dès le sixième carnet de captivité, à analyser sous l'angle de la "durée en tant qu'être » ${ }^{44}$ et à mettre en relation avec un des thèmes les plus centraux de sa philosophie, eros:

Analyse de la durée en tant qu'être - non pas ce qui reste sous le changement, car précisément en quoi consiste ce demeurer quand on ne veut plus le loger

41. Dans En découvrant l'existence avec Husserl et Heidegger, Paris, Vrin, 1967, p. 125-135.

42. Carnets de captivité, p. 147.

43. «Parole et Silence», p. 90.

44. Carnets de captivité, p. 167. 
dans les qualités de l'objet - ni bien entendu le changement, le périssable - ni le devenir - ni le présent - mais eros ou verbe d'être - ou son ${ }^{45}$.

Aussi la phénoménologie du son chez Levinas ne se présente-t-elle pas comme célébration ou défense de la subjectivité. Levinas n'a pas pour souci de donner substance au son qui serait le mouvement par lequel l'accomplissement s'accomplit, la durée dure, autrement dit, «le temps pur» ${ }^{46}$. Il a pour objectif d'opposer résolument objectivité et transcendance, projet qu'il esquisse dès la captivité et qu'il déploiera dans Totalité et Infini ${ }^{47}$. Mais comment parvenir à ne plus penser la transcendance comme savoir? C'est dans le concept temporel d'immédiateté que Levinas puisera, à l'encontre de l'immédiateté husserlienne, l'expérience sensible d'un corps propre comme exposition du sens. L'immédiateté du son dont parle Levinas est bien le fait d'une signification sans contexte. Le son sonne, der Klang Klingelt, en dehors de toute donation de sens. C'est donc à partir de la sensation, depuis le noumène que Levinas soustrait l'expérience sensible du son au solipsisme dans lequel il tend à retomber. Cette transcendance pour Levinas,

[...] autre que celle du pouvoir et de l'intellection qui se fait par projection - c'est précisément l'expression. Ce n'est pas la lumière qui est ici le prototype sensible, mais le son. La relation avec l'être, dans sa gloire d'être - c'est entendre ${ }^{48}$.

Un peu plus loin dans le texte, Levinas poursuit sa réflexion sur la sortie de la lumière par le son, une évasion pour le moins singulière et inattendue qui est tout le contraire d'une échappée par l'ouverture d'une fenêtre par exemple, car le son ne s'annonce pas, pas plus qu'il annonce «à celui qui l'entend quelque chose qui peut-être englobé dans sa solitude» ${ }^{49}$. La fonction annonciatrice du son est tournée vers l'avenir, tournée vers «la gloire d'un autre être ${ }^{50}$. D'où l'idée que la qualité la plus essentielle du son est de se déverser dans le temps. Qualité prophétique et eschatologique?

Pour illustrer cette conception absolument neuve du son, Levinas se réfère à un poème de Pouchkine intitulé «Le Prophète». Dans cette poésie, se produit une transmutation du sens qui mène à la connaissance prophétique. Mais cette transformation transite par l'oreille, elle

45. Ibid.

46. Ibid., p. 82.

47. La Haye, Martinus Nijhoff, 1961.

48. "Parole et Silence», p. 89.

49. Ibid., p. 90.

50. Ibid. 
[...] étend graduellement l'ouïe jusqu'à la perception de l'événement même

de l'être, comme si l'être même des choses pouvait être entendu:

«Il a effleuré mes oreilles

Et elles se remplirent de bruits et de sons

Et j'ai entendu la contradiction des cieux

Et le vol des anges qui montaient

Et la marche des monstres sous-marins

Et la croissance de la branche dans la vallée ${ }^{51}$.

Non seulement le son annonce la gloire de l'événement, mais il est aussi parole poétique sur cet événement, et en ce sens, il est mystère: «le mystérieux de l'être en tant qu'autre ${ }^{52}$. Le passage du son comme vibration de l'être au son comme événement de la langue n'est pas incongru sous la plume de Levinas. Nous savons que pour lui la langue ne désigne pas uniquement la chose, de même le son ne désigne pas nécessairement le signe. Nous comprenons pourquoi Levinas écrit que «Le mot n'est pas seulement le nom d'un objet ou d'une idée, il est le retentissement de l'être du sujet " ${ }^{53}$. Un symbole donc, dissocié de la chose et du signe et par conséquent, « comme un appel de l'au-delà " 54 . C'est encore à la cloche que Levinas songe lorsqu'il fait référence dans «Parole et Silence» à l'au-delà. Rappelons-nous ce qu'il écrivait dans les Carnets de captivité: cette cloche, dont la vocation prophétique est de crever le monde de la lumière, «remplit l'espace de quelque chose qui vient de là-bas ${ }^{55}$.

Au-delà et là-bas: ces deux occurrences sont dans un rapport de parenté. Elles marquent un moment de ce que Levinas appelle "le goût du temps» ${ }^{56}$. Levinas fait remonter la phénoménologie du son au bord d'un impensé qui rompt avec l'ordre du voir. Mais la séparation entre la lumière et le son, entre le voir et l'entendre, entre la vue et l'ouïe est d'un autre ordre que celui de la distance et sa signification est antérieure à l'intelligibilité. Avec le son, le monde se rompt et le temps aussi. Il accroît les défaillances du langage tout en se rendant effectif comme Verbe. C'est le moment où il devient musique, le moment dit Levinas où «il est intégré dans un ensemble, [...] La sonorité dans son ensemble, décrit la structure d'un monde où l'autre peut apparaître " ${ }^{57}$. L'entrée dans le son est entrée dans

51. "Parole et Silence», p. 91-92.

52. Ibid., p. 92.

53. Ibid.

54. Ibid., p. 93.

55. Carnets de captivité, p. 152.

56. Ibid.

57. «Parole et Silence», p. 93. 
le monde et entrée dans l'être qui chez Levinas entre en opposition avec la connaissance, la substance. De sorte que, pareille à l'œuvre littéraire où pour Levinas «un monde créé ne vaut comme le monde réel lui-même, que par le surnaturel auquel il laisse la marge et qu'il fait pressentir", le son comme manifestation du au-delà et du là-bas fait retentir l'être de manière quasi surnaturelle. Ce qu'il dit de lui, ce qu'il raconte a d'ores et déjà perdu sa signification puisque sa signification lui vient du dehors: «[...] si la fonction première du mot consiste dans ce retentissement de l'être, il doit être possible d'en déduire la modification essentielle du son en mot ${ }{ }^{8}$. La modification du son en mot est à entendre comme instant surnaturel du passage de l'implicite à l'explicite.

\section{"Le verbe c'est le son " 59 : l'inclinaison érotique}

Repartons de ce postulat et demandons-nous comment Levinas articule l'extrême matérialité du son, sa texture vibratoire depuis laquelle la musique se temporalise avec l'idée que la "sonorité du son» viendrait féconder le temps du monde dans la potentialité de chacun de ses instants d'éclat. Je note au passage que la matérialité du son telle que la décrit Levinas rappelle singulièrement la dimension de terrestrialité (Erdhaftigkeit) que Heidegger entendait dans L'origine de l'œuvre d'art ${ }^{60}$, comme étant un paramètre constitutif de l'œuvre, ne s'offrant pas à la révélation du monde.

Il y a là chez Levinas un puissant paradoxe, à l'origine dans les Carnets de captivité puis dans la conférence «Parole et Silence» d'une méditation sur la nécessité malgré tout de s'évader de l'écoute sonore synonyme de verbalité de l'être, de s'échapper de ce paroxysme de matérialité lumineuse et formelle qui serait une autre manière de dire que l'on est ontiquement au plus proche de soi-même. Cependant que cette proximité avec soi-même conduit également Heidegger à dire: "Je suis ontologiquement le plus éloigné de moi-même».

Deux priorités donc, ontique et ontologique, se partageraient la réflexion lévinassienne sur la phénoménologie du son, à laquelle viendrait s'ajouter une troisième priorité qui affecte le caractère social et érotique du son. Puisque le signe se présente comme un renvoi à ce qui est absent, à savoir la lumière, le son « au lieu de manifester la plénitude de l'exister - l'exprime en renvoyant à autre chose qui la subjectivise ${ }^{61}$. Cet "autre chose», événement dans l'être, Levinas le relie à l'irréductibilité de la relation sociale

58. Ibid., p. 92.

59. Carnets de captivité, p. 166.

60. Dans Chemins qui ne mènent nulle part, trad. fr. W. Brokmeir, Paris, Gallimard, 1962.

61. «Parole et Silence», p. 99. 
qui passe de la représentation à autrui à «la relation érotique ${ }^{62}$. Le son est donc «scandale» non seulement parce qu'il interrompt le bruissement ininterrompu de l'être, mais parce qu'il est évasion de soi dans la relation érotique qui annonce l'avenir.

D'un côté, le son est pensé comme doublure de la chose; de l'autre, il n'est rien d'autre que lui-même, il «n'est rien ${ }^{63}$, écrit Levinas, rien, c'est-à-dire qu'il ne précède pas le langage. Il s'affirme phénoménalement, comme une entité qui n'appartient plus au monde solitaire de la lumière, si grand soit-il.

Ce n'est donc pas un défaut, mais un avantage du son, que de déborder sa forme et de ne pas nous donner un autre monde qui en tant que lumière serait englobé dans celui où nous sommes ${ }^{64}$.

Dès la captivité, et même avant, Levinas aura donc cherché à renouveler le langage philosophique, lequel lui sembler étouffer sous le poids mortifère de la métaphysique. Mais le langage métaphysique aura également relié la parole au son comme nous l'avons vu avec Hegel, plus exactement avec l'expression sonore, laquelle serait la manifestation des affects purs qui demeurent étroitement soudés au phénomène de la vision. Chez Hegel, l'âme se sert de la musique pour émouvoir les âmes, pour les faire chavirer. Dans les Carnets de captivité, Levinas écrit que:

L'âme est dans les yeux. [...] Mais regarder dans les yeux, c'est regarder se regarder, plus encore c'est: je regarde me regarder regardant me regarder... Itération à l'infini réalisée dans l'instant. [...] C'est pourquoi primat de la vision. Pour l'ouie, pour le toucher rien de tel ${ }^{65}$.

Levinas souligne bien ici sans le mentionner une propriété du son qui tend à exprimer un chez soi structurant l'extériorité de l'intention elle-même. Cette intention donne le ton. Les Grecs disaient qu'elle donne le mode. Or chez Heidegger, les tons sont quasiment des images spectrales, ils ne font

qu'apparaître puis disparaître dans l'espace vide de l'âme. Mais le Dasein est toujours, en tant que Dasein, déjà de fond en comble accordé à un ton. Ce qui a lieu, c'est un changement incessant de tonalités ${ }^{66}$.

62. "Parole et Silence», p. 97.

63. Ibid., p. 92.

64. Ibid., p. 90.

65. Carnets de captivité, p. 104.

66. Les concepts fondamentaux de la métaphysique. Monde - Finitude - Solitude, texte établi par F.W. von Hermann, traduit par D. Panis, Paris, Gallimard, 1992, p. 109. 
Contrairement au ton trop subordonné à la figure, au Leitmotiv, à la Stimmung qui induit la notion d'âme, le son chez Levinas instaure plus qu'un rythme entre chaque battement du son, plus qu'un intervalle. Il instaure une multiplicité d'instants qui ne sont pas l'accomplissement d'un acte mais l'acte lui-même. C'est en ce sens qu'il faut comprendre que le son est déjà en soi un paradigme de rupture, un drame, un affect profondément altéré par ce qui déborde la qualité sensible par elle-même du son. Alors que la vision qui donne accès à l'âme, c'est-à-dire à la lumière, ne se donne pas comme surplus qui s'extériorise et s'épanche vers son avenir, mais comme surplus qui contemple son supplément d'âme, qui écoute sa propre tonalité, qui s'enroule dans sa Stimmung.

Le son est donc essentiellement temps. Non plus «singulière tragédie ${ }^{67}$ que nous éprouvons au fond de l'ennui d'être, mais drame, en ce sens qu'il ne vise pas l'effectuation de la vérité. Il est temps et drame, temps comme drame, parce qu'en doublant toutes les manifestations de la chose par un surplus d'expression qui est l'inverse d'un «se regarder dans les yeux", le son devient une modalité du temps qui n'a d'existence propre que parce qu'il dure. Cette doublure du son qui signale la part d'événement dans les manifestations de l'être dont le site est celui du rassemblement (Versammlung) qui a pour essence le retrait (Abgeschiedenheit), Levinas cherche à la desceller. Que la relation avec autrui soit ce dont elle sonne et que ce dont elle sonne soit ce dont on parle avant toute thématisation, voilà bien le lieu d'une inclinaison érotique qui, chez Levinas, s'ouvrira à l'unicité de l'unique. De fait, la sonorité du son, dont la vocation est de percer le monde continu de la lumière, décrit la structure éthique de la subjectivité dans son rapport étroit à l'équivocité d'éros ${ }^{68}$. Question sur laquelle nous reviendrons.

Danielle CoHen-Levinas

Université Paris-Sorbonne, Paris IV

67. "Parole et Silence», p. 96.

68. Le lien entre la phénoménologie du son et éros est développé dans un autre texte intitulé «Eros, transcendance et fécondité», rédigé pour le colloque international autour des cinquante ans de Totalité et Infini, organisé par Alexander Schnell et Danielle Cohen-Levinas, Université Paris IV Sorbonne et Les Archives Husserl - ENS de Paris, 18 et 19 novembre 2011. 